\title{
Predictors of Long-Term Pain After Hip Arthroplasty in Patients With Femoral Neck Fractures: A Cohort Study
}

\author{
Atefeh Noori, MSc, PhD (Cand), ${ }^{a, b}$ Sheila Sprague, PhD, ${ }^{a, c}$ Sofia Bzovsky, MSc, ${ }^{c}$ \\ Emil H. Schemitsch, MD, FRCSC, ${ }^{d}$ Rudolf W. Poolman, MD, PhD, ${ }^{e}$ Frede Frihagen, MD, ${ }^{f}$ \\ Daniel Axelrod, MD, MSc (Cand), ${ }^{c}$ Diane Heels-Ansdell, MSc, ${ }^{a}$ Mohit Bhandari, MD, PhD, FRCSC, ${ }^{a, c}$ \\ and Jason W. Busse, DC, PhD ${ }^{a, b, g, h}$ on behalf of the HEALTH Investigators
} Objectives: To identify factors associated with the development of
prolonged pain after hip fracture surgery.

Design: Secondary analysis of a randomized controlled trial.

Setting: Eighty hospitals in 10 countries.

Patients/Participants: One thousand four hundred forty-one hip fracture patients in the HEALTH trial.

Interventions: Total hip arthroplasty or hemiarthroplasty.

Main Outcome Measures: Moderate-to-severe pain (at least 2 activities on the Western Ontario and McMaster Universities Osteoarthritis questionnaire pain subscale with scores $\geq 2$ ) at 12 and 24 months after hip arthroplasty.

Results: Of 840 and 726 patients with complete baseline data and outcomes at 1-year and 2-year follow-up, 96 (11.4\%) and 80 $(11.0 \%)$ reported moderate-to-severe pain, respectively. An increased risk of pain at both 1 and 2 years after surgery was associated with reporting moderate-to-severe hip pain before fracture [absolute risk increase (ARI) $15.3 \%$, 95\% confidence interval (CI) 6.44\%-24.35\%; ARI 12.5\%, 95\% CI 2.85\%-22.12\%, respectively] and prefracture opioid use (ARI 15.6\%, 95\% CI $5.41 \%-25.89 \%$; ARI $21.1 \%$; 95\% CI $8.23 \%-34.02 \%$, respectively). Female sex was associated with an increased risk of persistent pain at 1 year (ARI $6.2 \%, 95 \%$ CI $3.53 \%-8.84 \%$ ). A greater risk of persistent pain at 2 years was associated with younger age (s79-year-old; ARI 6.3\%; 95\% CI $2.67 \%-9.91 \%$ ) and higher prefacture functional status (ARI 10.7\%; 95\% CI $3.80 \%-17.64 \%)$.

Conclusions: Among hip fracture patients undergoing arthroplasty, approximately one in 10 will experience moderate-to-severe pain up to 2 years after surgery. Younger age, female sex, higher functioning prefracture, living with hip pain prefracture, and use of prescription opioids were predictive of persistent pain.

Key Words: hip arthroplasty, hemiarthroplasty, femoral neck fracture, predictors, pain

Accepted for publication August 11, 2020 .

From the ${ }^{\mathrm{a}}$ Department of Health Research Methods, Evidence, and Impact, McMaster University, Hamilton,Ontario, Canada; ${ }^{\mathrm{b}}$ The Michael G. DeGroote National Pain Center, McMaster University, Hamilton, ON, Canada; ' Division of Orthopaedic Surgery, Department of Surgery, McMaster University, Hamilton, ON, Canada; ${ }^{\mathrm{d} D e p a r t m e n t}$ of Surgery, University of Western Ontario, London, ON, Canada; ${ }^{\mathrm{e} D e p a r t m e n t ~ o f ~ O r t h o p e d i c ~ a n d ~ T r a u m a ~ S u r g e r y, ~ O L V G, ~ A m s t e r d a m ~}$ and Leiden University Medical Center, Leiden, the Netherlands; ${ }^{\mathrm{f}}$ Division of Orthopaedic Surgery, Oslo University Hospital, Oslo, Norway; ${ }^{\mathrm{g} D e p a r t m e n t}$ of

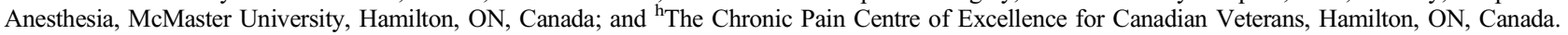

The HEALTH trial was supported by research grants from the Canadian Institutes of Health Research (CIHR) (MCT-90168), National Institutes of Health (NIH) (1UM1AR063386-01), ZorgOnderzoek Nederland-medische wetensehappen (ZonMw) (17088.2503), Sophies Minde Foundation for Orthopaedic Research, McMaster Surgical Associates, and Stryker Orthopaedics. The funding sources had no role in design or conduct of the study; the collection, management, analysis, or interpretation of the data; or the preparation, review, or approval of the manuscript.

S. Sprague reports editorial or governing board for BMS Women's Health, employment from Global Research Solutions Inc, and employment from McMaster University, outside the submitted work. E. H. Schemitsch reports personal fees from Acumed, LLC, personal fees from Amgen Co, research support from Biocomposites, board or committee member for the Canadian Orthopaedic Association, personal fees from DePuy, board or committee member for the Hip Society, board or committee member for the International Society for Fracture Repair, personal fees from ITS, editorial or governing board for the Journal of Orthopaedic Trauma, board or committee member for the Orthopaedic Trauma Association, editorial or governing board for the Orthopaedic Trauma Association International, board or committee member for the Osteosynthesis and Trauma Care Foundation, personal fees from Pentopharm, personal fees from Sanofi-Aventis, personal fees from Saunders/Mosby-Elsevier, personal fees from Smith \& Nephew, personal fees from Springer, personal fees from Stryker, personal fees from Swemac, and personal fees from Zimmer, outside the submitted work. R. W. Poolman reports board or committee member for the Dutch Orthopaedic Association, research support from Lima, and research support from Link Orthopaedics, outside the submitted work. F. Frihagen reports personal fees from Amgen Co, personal fees from Smith \& Nephew, personal fees from Synthes, and personal fees from Zimmer, outside the submitted work. M. Bhandari reports research support from Acumed, LLC, research support from Aphria, research support from Ferring Pharmaceuticals, research support and personal fees from Pendopharma, and research support and personal fees from Sanofi-Aventis, outside the submitted work. The remaining authors report no conflict of interest.

Supplemental digital content is available for this article. Direct URL citations appear in the printed text and are provided in the HTML and PDF versions of this article on the journal's Web site (www.jorthotrauma.com).

Reprints: Jason W. Busse, DC, PhD, Michael G. DeGroote National Pain Centre, McMaster University, HSC-2V9, 1280 Main St. West, Hamilton, ON, Canada L8S 4K1 (e-mail: bussejw@mcmaster.ca).

Copyright (C) 2020 Wolters Kluwer Health, Inc. All rights reserved.

DOI: 10.1097/BOT.0000000000001929 
Level of Evidence: Prognostic Level II. See Instructions for Authors for a complete description of levels of evidence.

(J Orthop Trauma 2020;34:S55-S63)

\section{INTRODUCTION}

Hip fracture is a severe and frequent event affecting a large number of older adults around the world. ${ }^{1}$ Approximately 52,000 hip fracture-related hospital admissions were reported among the population aged 80 years and older between 2011 and 2015 in Ontario, Canada, alone. ${ }^{2}$ Hip fractures are associated with impaired mobility, loss of quality of life, and one-year mortality rate estimations between $14 \%$ and $58 \%$ after injury. ${ }^{3-7}$

Displaced femoral neck fractures are common injuries, which are often managed with arthroplasty, especially for patients $\geq 80$ years. $^{8}$ With an aging population globally, the demands for arthroplasty are predicted to increase substantially. ${ }^{9}$ Although symptom relief is an important goal of hip arthroplasty, many patients experience persistent postsurgical pain. ${ }^{10}$ For instance, one study of patients with a displaced subcapital hip fracture found that $13 \%$ reported moderate-to-severe persistent pain at one year after the surgery. ${ }^{11}$ Some evidence suggests that total hip arthroplasty (THA) may provide better long-term pain relief than hemiarthroplasty ${ }^{12}$; however, the HEALTH randomized controlled trial results did not find a benefit of THA over hemiarthroplasty in reducing persistent pain. ${ }^{13}$ Improved understanding of factors associated with long-term pain after hip arthroplasty could facilitate targeting of high-risk patients in an effort to improve prognosis.

In this study, we aimed to investigate which factors (modifiable and nonmodifiable) are associated with moderateto-severe pain at 12 and 24 months after hip arthroplasty in participants aged 50 years and older with a displaced femoral neck fracture.

\section{MATERIAL AND METHODS}

\section{Participants}

This study used data from 1441 participants enrolled in the HEALTH trial, a randomized controlled trial that explored the effect of THA or hemiarthroplasty for patients $\geq 50$ years with a displaced femoral neck fracture. ${ }^{13}$

\section{Outcome Measure}

In the HEALTH trial, the Western Ontario and McMaster Universities Osteoarthritis (WOMAC) questionnaire was administered to assess hip-related pain status of patients before their fracture occurred (completed at time of study enrollment) and at 12 months and 24 months after surgery. The WOMAC has been shown to be valid, reliable, responsive, and feasible in hip fracture patients. ${ }^{14}$ The questionnaire contains 5 different activity questions for pain intensity assessment with options of "no," "mild," "moderate," "severe," and "extreme" pain. We summed these scores for all 5 questions and calculated the overall pain score (range 020) for each patient. In keeping with previous studies, we defined our outcome as the presence of moderate-to-severe pain (a score of 2 or more for at least 2 questions on activities using the WOMAC pain subscale) or no moderate-to-severe pain (a score of 0 or 1 for at least 3 items on activities, which we used as the reference group in our analysis) at 12 and 24 months after hip surgery. ${ }^{15-17}$

\section{Predictors}

The first group of predictors consisted of demographic variables, including age ( $\leq 79$ vs. $>79$ years. 79 was the median age of all included participants) and sex (women vs. men). We also considered prefracture variables including those reporting moderate-to-severe hip pain before the occurrence of the fracture (yes vs. no), use of opioid medications before fracture (yes vs. no), prefracture functional status (ambulating without assistance vs. use of an assistive device), prefracture living status (institutionalized vs. noninstitutionalized), previous surgery affecting hip (yes vs. no), having major comorbidities including back pain, osteoarthritis, cancer, rheumatoid arthritis, and depression (yes vs. no), body mass index (BMI, $25-34.9 \mathrm{~kg} / \mathrm{m}^{2}$ vs. $\leq 24.9 \mathrm{~kg} / \mathrm{m}^{2}$ ), and the American Society of Anesthesiologists (ASA) classification (III/IV/V vs. I/II). In addition, procedure-related factors, including type of arthroplasty surgery (THA vs. bipolar or unipolar hemiarthroplasty) and type of surgical approach (anterolateral/direct anterior vs. posterior/posterolateral), were included. We also considered 4 postoperative variables, all of which were measured up to 30 days after surgery, including patient's weight-bearing status (non/partial vs. full), use of any physiotherapy or rehabilitation (yes vs. no), serious adverse events (yes vs. no), and fracture-related complications (complication with or without revision surgery vs. no complication). We chose 30 days after surgery as our cut-off mark to avoid concerns regarding the temporality of the predictors and the study outcomes.

\section{Statistical Analysis}

We reported the mean and SD of continuous variables, and absolute and relative frequencies for categorical variables. We used logistic regression analyses to determine predictors of moderate-to-severe pain at 12 and 24 months after hip fracture repair. We excluded patients from our analyses if their pain score, measured using the WOMAC, before fracture and at follow-up times (12 and 24 months), were unavailable. We first ran univariable models to estimate the unadjusted odds ratios (ORs), then we constructed multivariable models for both visits. We selected 12 independent factors for multivariable models previously reported as important predictors ${ }^{18}$ or variables judged to be associated with persistent pain by experts, including age, sex, BMI, prefracture moderate-to-severe hip pain, opioid use, functional status, depression, weight-bearing status, use of any physiotherapy or rehabilitation, and serious adverse events. We also adjusted for the type of arthroplasty surgery and surgical approach that were used in the HEALTH trial. We excluded independent variables with fewer than 50 observations per category, unless we were able to collapse them with other related variables to exceed this threshold (Table 1). We then applied the Hosmer-Lemeshow (H-L) goodness of fit test for these adjusted models ${ }^{19}$ and reported the statistics. 
TABLE 1. Demographics and Clinical Characteristics at 12- and 24-Month Follow-up Visits in Patients With Femoral Neck Fractures

\begin{tabular}{|c|c|c|c|c|c|c|}
\hline \multirow[b]{2}{*}{ Characteristics } & \multicolumn{3}{|c|}{12 months } & \multicolumn{3}{|c|}{24 months } \\
\hline & $\begin{array}{c}\text { Total, } \\
\mathbf{n}=\mathbf{8 4 0}\end{array}$ & $\begin{array}{c}\text { No Moderate-to- } \\
\text { Severe Pain, } n=744\end{array}$ & $\begin{array}{l}\text { Moderate-to-Severe } \\
\text { Pain, } \mathrm{n}=96\end{array}$ & $\begin{array}{c}\text { Total, } \\
\mathrm{n}=\mathbf{7 2 6}\end{array}$ & $\begin{array}{c}\text { No Moderate-to- } \\
\text { Severe Pain, } n=646\end{array}$ & $\begin{array}{c}\text { Moderate-to-Severe } \\
\text { Pain, } \mathbf{n}=\mathbf{8 0}\end{array}$ \\
\hline \multicolumn{7}{|l|}{ Demographic factors } \\
\hline \multicolumn{7}{|l|}{ Age, n (\%) } \\
\hline $\begin{array}{l}\text { Below median } \\
\text { ( } \leq 79 \text { years })\end{array}$ & $399(47.5)$ & $346(86.7)$ & $53(13.3)$ & $362(49.9)$ & $315(87.0)$ & $47(13.0)$ \\
\hline $\begin{array}{l}\text { Above median } \\
(>79 \text { years })\end{array}$ & $441(52.5)$ & $398(90.2)$ & $43(9.8)$ & $364(50.1)$ & $331(91.0)$ & $33(9.0)$ \\
\hline \multicolumn{7}{|l|}{ Sex, n (\%) } \\
\hline Female & $618(73.6)$ & $538(87.0)$ & $80(13.0)$ & $546(75.0)$ & $484(88.6)$ & $62(11.3)$ \\
\hline Male & $222(26.4)$ & $206(92.8)$ & $16(7.2)$ & $180(25.0)$ & $162(90.0)$ & $18(10.0)$ \\
\hline \multicolumn{7}{|l|}{ Preoperative factors } \\
\hline \multicolumn{7}{|l|}{ BMI, n (\%) } \\
\hline$\leq 24.9$ & $431(52.0)$ & $384(89.1)$ & 47 (10.9) & $368(51.0)$ & $332(90.0)$ & $36(10.0)$ \\
\hline $25-34.9$ & $399(48.0)$ & $352(88.2)$ & $47(11.8)$ & $351(49.0)$ & $308(88.0)$ & $43(12.0)$ \\
\hline \multicolumn{7}{|l|}{$\begin{array}{l}\text { Prefracture moderate- } \\
\text { to-severe hip pain, } \\
\mathrm{n}(\%)^{*}\end{array}$} \\
\hline No & $696(89.7)$ & $631(90.7)$ & $65(9.3)$ & $605(90.7)$ & $552(91.2)$ & $53(8.8)$ \\
\hline Yes & $80(10.3)$ & $60(75.0)$ & $20(25.0)$ & $62(9.3)$ & $45(72.6)$ & $17(27.4)$ \\
\hline \multicolumn{7}{|l|}{$\begin{array}{l}\text { Prefracture opioid use, } \\
\text { n (\%) }\end{array}$} \\
\hline No & $774(92.1)$ & $695(89.8)$ & $79(10.2)$ & $670(92.2)$ & 607 (90.6) & $63(9.4)$ \\
\hline Yes & $66(7.9)$ & 49 (74.2) & $17(25.8)$ & $56(7.7)$ & $39(69.6)$ & $17(30.3)$ \\
\hline \multicolumn{7}{|l|}{$\begin{array}{l}\text { Prefracture functional } \\
\text { status, } \mathrm{n}(\%)\end{array}$} \\
\hline $\begin{array}{l}\text { Uses an assistive } \\
\text { device }\end{array}$ & $673(80.1)$ & $603(89.6)$ & $70(10.4)$ & $589(81.1)$ & $535(91)$ & $54(9)$ \\
\hline $\begin{array}{l}\text { Ambulate without } \\
\text { assistance }\end{array}$ & 167 (19.9) & $141(84.4)$ & $26(15.6)$ & $137(18.8)$ & $111(81)$ & $26(19)$ \\
\hline \multicolumn{7}{|l|}{$\begin{array}{l}\text { Prefracture living } \\
\text { status, } \mathrm{n}(\%) \dagger\end{array}$} \\
\hline Not institutionalized & 822 (97.9) & $731(89.0)$ & $91(11.0)$ & 717 (98.7) & $639(89.1)$ & $78(10.8)$ \\
\hline Institutionalized & $18(2.1)$ & $13(72.2)$ & $5(27.8)$ & $9(1.2)$ & 7 (77.7) & $2(22.2)$ \\
\hline \multicolumn{7}{|l|}{$\begin{array}{l}\text { Previous surgery to } \\
\text { affected hip n (\%)† }\end{array}$} \\
\hline No & $836(99.6)$ & $740(88.5)$ & $96(11.5)$ & 723 (99.7) & $644(89.0)$ & $79(10.9)$ \\
\hline Yes & $3(0.4)$ & $3(100)$ & $0(0)$ & $2(0.28)$ & $1(50.0)$ & $1(50.0)$ \\
\hline \multicolumn{7}{|l|}{ ASA class, n (\%) } \\
\hline I/II & $416(49.5)$ & $370(89.0)$ & $46(11.0)$ & $389(53.5)$ & $354(91)$ & $35(9)$ \\
\hline III/IV/V & $424(50.4)$ & $374(88.2)$ & $50(11.8)$ & $337(46.4)$ & $292(87)$ & $45(13)$ \\
\hline \multicolumn{7}{|l|}{$\begin{array}{l}\text { Preoperative } \\
\text { comorbidities }\end{array}$} \\
\hline \multicolumn{7}{|l|}{$\begin{array}{l}\text { Rheumatoid arthritis, } \mathrm{n} \\
\quad(\%)\end{array}$} \\
\hline No & $817(97.3)$ & $725(88.8)$ & $92(11.2)$ & 704 (96.9) & $627(89)$ & $77(11)$ \\
\hline Yes & $23(2.7)$ & $19(82.6)$ & $4(17.4)$ & $22(3.0)$ & $19(86)$ & $3(14)$ \\
\hline \multicolumn{7}{|l|}{ Osteoarthritis, n (\%) } \\
\hline No & $714(85)$ & $643(90.0)$ & $71(10.0)$ & $623(85.8)$ & $556(90)$ & $67(10)$ \\
\hline Yes & $126(15)$ & $101(80.1)$ & $25(19.9)$ & $103(14.1)$ & $90(87)$ & $13(13)$ \\
\hline \multicolumn{7}{|l|}{ Cancer, n (\%) } \\
\hline No & $757(90.1)$ & $674(89.0)$ & $83(11.0)$ & $654(90.0)$ & $583(89)$ & $71(11)$ \\
\hline Yes & $83(9.9)$ & $70(84.3)$ & $13(15.7)$ & $72(9.9)$ & $63(87.5)$ & $9(12.5)$ \\
\hline
\end{tabular}


TABLE 1. (Continued) Demographics and Clinical Characteristics at 12- and 24-Month Follow-up Visits in Patients With Femoral Neck Fractures

\begin{tabular}{|c|c|c|c|c|c|c|}
\hline \multirow[b]{2}{*}{ Characteristics } & \multicolumn{3}{|c|}{12 months } & \multicolumn{3}{|c|}{24 months } \\
\hline & $\begin{array}{c}\text { Total, } \\
\mathrm{n}=\mathbf{8 4 0}\end{array}$ & $\begin{array}{c}\text { No Moderate-to- } \\
\text { Severe Pain, } n=744\end{array}$ & $\begin{array}{c}\text { Moderate-to-Severe } \\
\text { Pain, } \mathbf{n}=96\end{array}$ & $\begin{array}{c}\text { Total, } \\
n=726\end{array}$ & $\begin{array}{c}\text { No Moderate-to- } \\
\text { Severe Pain, } n=646\end{array}$ & $\begin{array}{c}\text { Moderate-to-Severe } \\
\text { Pain, } \mathbf{n}=\mathbf{8 0}\end{array}$ \\
\hline \multicolumn{7}{|l|}{ Depression, n (\%) } \\
\hline No & $738(87.9)$ & $658(89.1)$ & $80(10.9)$ & $639(88.0)$ & $570(89)$ & $69(11)$ \\
\hline Yes & $102(12.1)$ & $86(84.0)$ & $16(16.0)$ & 87 (11.9) & $76(87)$ & $11(13)$ \\
\hline \multicolumn{7}{|l|}{ Back pain, n (\%) } \\
\hline No & $759(90.3)$ & $679(89.4)$ & $80(10.6)$ & $655(90.2)$ & $594(91)$ & $61(9)$ \\
\hline Yes & $81(9.7)$ & $65(80.2)$ & $16(19.8)$ & $71(9.7)$ & $52(73)$ & $19(27)$ \\
\hline \multicolumn{7}{|l|}{ Procedure-related factors } \\
\hline \multicolumn{7}{|l|}{$\begin{array}{l}\text { Surgical approach, } \\
\text { n }(\%)\end{array}$} \\
\hline $\begin{array}{l}\text { Posterior/ } \\
\text { posterolateral }\end{array}$ & $315(37.5)$ & $284(90.1)$ & $31(9.9)$ & $280(38.6)$ & $249(89)$ & $31(11)$ \\
\hline $\begin{array}{l}\text { Anterolateral/direct } \\
\text { anterior }\end{array}$ & $524(62.46)$ & $459(87.6)$ & $65(12.4)$ & $446(61.4)$ & $397(89)$ & $49(11)$ \\
\hline \multicolumn{7}{|l|}{ Surgery type, n (\%) } \\
\hline THA & $414(49.4)$ & $372(89.9)$ & $42(10.1)$ & $363(50.0)$ & $331(91.2)$ & $32(8.8)$ \\
\hline $\begin{array}{l}\text { Bipolar } \\
\text { hemiarthroplasty }\end{array}$ & $212(25.3)$ & $186(87.8)$ & $26(12.2)$ & $189(26.0)$ & $164(86.8)$ & $25(13.2)$ \\
\hline $\begin{array}{l}\text { Unipolar } \\
\text { hemiarthroplasty }\end{array}$ & $212(25.3)$ & $184(86.8)$ & $28(13.2)$ & $173(23.8)$ & $150(86.8)$ & $23(13.2)$ \\
\hline \multicolumn{7}{|l|}{ Postoperative factors } \\
\hline \multicolumn{7}{|l|}{$\begin{array}{l}\text { Early weight-bearing } \\
\text { status, n }(\%) \ddagger\end{array}$} \\
\hline Non or partial & $355(42.2)$ & $317(89.3)$ & $38(10.7)$ & $295(40.7)$ & $258(87.4)$ & $37(12.6)$ \\
\hline Full & $485(57.8)$ & $427(88.0)$ & $58(12.0)$ & $431(59.3)$ & $388(90.0)$ & $43(10.0)$ \\
\hline \multicolumn{7}{|l|}{$\begin{array}{l}\text { Early physiotherapy or } \\
\text { rehabilitation, } \\
\mathrm{n}(\%) \ddagger\end{array}$} \\
\hline No & $61(7.3)$ & $51(83.6)$ & $10(16.4)$ & $53(7.3)$ & $44(83.0)$ & $9(17.0)$ \\
\hline Yes & $774(92.7)$ & $690(89.1)$ & $84(10.9)$ & $669(92.7)$ & $599(89.5)$ & $70(10.5)$ \\
\hline \multicolumn{7}{|l|}{$\begin{array}{l}\text { Serious adverse events, } \\
\text { n }(\%) \S\end{array}$} \\
\hline No & $672(80.0)$ & $596(88.7)$ & $76(11.3)$ & $594(81.8)$ & $530(89.2)$ & $64(10.8)$ \\
\hline Yes & $168(20.0)$ & $148(88.1)$ & $20(11.9)$ & $132(18.2)$ & $116(87.9)$ & $16(12.1)$ \\
\hline \multicolumn{7}{|l|}{$\begin{array}{l}\text { Fracture-related } \\
\text { complications, } \\
\mathrm{n}(\%) \dagger \S\end{array}$} \\
\hline No complication & $774(92.2)$ & $686(88.6)$ & $88(11.4)$ & $673(92.7)$ & $600(89.2)$ & $73(10.8)$ \\
\hline $\begin{array}{l}\text { Complication } \\
\text { without revision } \\
\text { surgery }\end{array}$ & $29(3.4)$ & $27(93.1)$ & $2(6.9)$ & $22(3.0)$ & $19(86.4)$ & $3(13.6)$ \\
\hline $\begin{array}{l}\text { Complication with } \\
\text { revision surgery }\end{array}$ & $37(4.4)$ & $31(83.8)$ & $6(16.2)$ & $31(4.3)$ & $27(87.1)$ & $4(12.9)$ \\
\hline \multicolumn{7}{|c|}{$\begin{array}{l}\text { *The WOMAC questionnaire was administered and asked patients to indicate the severity of hip pain before their fracture occurred. } \\
\dagger \text { Variables with very low frequency in one subcategory were not included in the regression models. } \\
\text { †These variables were collected immediately after surgery. } \\
\text { §These variables were collected within } 30 \text { days after surgery. } \\
\text { ASA, American Society of Anesthesiologists; BMI, body mass index; THA, total hip arthroplasty. }\end{array}$} \\
\hline
\end{tabular}

We assessed the impact of influential observations by calculating the deviance residual and explored for multicollinearity among the independent variables using the variance inflation factor, with values greater than 5 indicating a possible issue regarding collinearity. ${ }^{20}$ Discriminability of the models was checked using the area under the curve statistic, with values between 0.7 and 0.8 indicating acceptable classification performance of a model. We reported adjusted ORs (aORs) along with their 95\% confidence intervals (95\% CIs). In addition, we reported the absolute risk increase (ARI) for each significant predictor in the selected adjusted models by estimating the baseline risk of outcome 
(for both 12- and 24-month follow-up) among patients who did not have any significant risk factors. We also tested for an interaction between opioid use and functional status, hypothesizing that patients who used opioids and used assistive devices to ambulate before fracture may be more likely to experience moderate-to-severe persistent pain. Variables with $P$ values less than 0.05 in the adjusted models were considered statistically significant. All analyses were performed in Stata version 15 .

\section{RESULTS}

At 12- and 24-month follow-up visits, 1127 and 1122 patients, respectively, were eligible for our study. At the 1year follow-up visit, $64(5.7 \%)$ and by 2 years postoperatively, $113(10 \%)$ participants had died. Of the 1063 and 1009 patients who were alive, 840 (20\% missing data) and 726 (28\% missing data) had follow-up data available for the analysis of pain at 12 and 24 months follow-up, respectively. Details regarding participant flow and the reasons for exclusion are provided in Fig. 1.

\section{Patient Characteristics}

At the 12-month follow-up visit, 96 of the 840 patients $(11.4 \%)$ and at 24 months, 80 of the 726 patients $(11.0 \%)$ reported moderate-to-severe pain. Patients had a median age of 79 years, most were women $(73.0 \%$ at 12 months and $75.0 \%$ at 24 months), not institutionalized (approximately $98.0 \%$ at both visits) before the fracture occurred and did not have a previous surgery affecting the hip (>99.0\%). Demographic, preoperative/prefracture factors, comorbidities, procedure related, and postoperative characteristics among patients with and without moderate-to-severe pain are presented in Table 1.

\section{Multivariable Logistic Model at 12-Month Follow-Up Visit}

Among 165 patients without identified risk factors for pain (significant predictors in the adjusted model that reduced the risk of moderate-to-severe pain at 12-month visit), 8 reported moderate-to-severe pain at 1 year for a baseline risk of $4.8 \%$. In the adjusted model (Table 2 ), female patients (OR 1.85; 95\% CI 1.0-3.45; ARI 6.2\%, 95\% CI 3.53\%-8.84\%;
FIGURE 1. Cohort of patients' selection flow.

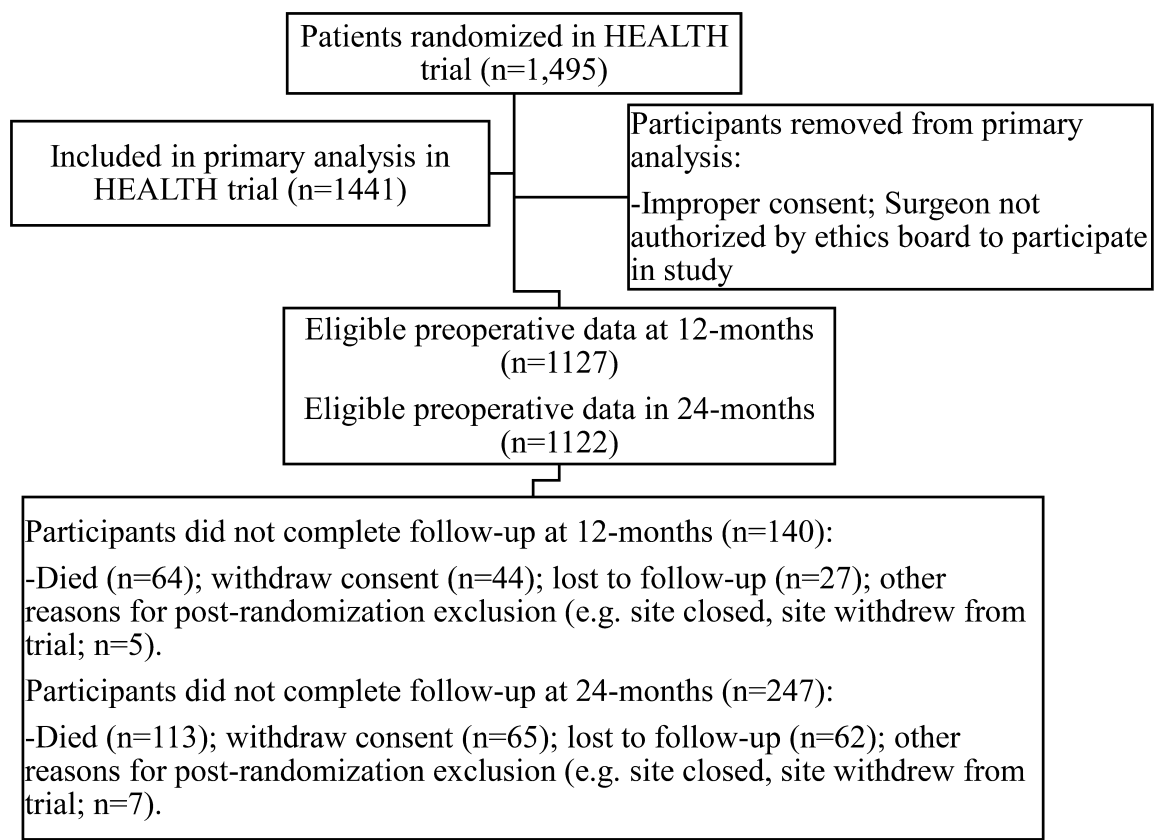

Patients who excluded from analysis due to missing outcome data at 12-months $(\mathrm{n}=147)$

Patients who excluded from analysis due to missing outcome data at 24-months $(n=149)$

Patients who were alive with complete data at 12-months $(\mathrm{n}=840)$

Patients who were alive with complete data at 24-months $(n=726)$ 
$P=0.049)$, patients with prefracture hip pain (OR 2.68; $95 \%$ CI 1.46-4.90; ARI 15.4\%, 95\% CI 6.44\%-24.35\%; $P=$ 0.001 ), and opioid use (OR 2.66; 95\% CI 1.35-5.24; ARI $15.7 \%$, 95\% CI $5.41 \%-25.90 \% ; P=0.005)$ had a significantly higher risk of experiencing moderate-to-severe pain at 1-year after surgery (Table 2).

\section{Multivariable Logistic Model at 24-Month Follow-Up Visit}

Among 218 patients without identified risk factors for pain (significant predictors in the adjusted model that reduced the risk of moderate-to-severe pain at 24-months visit), 11 reported moderate-to-severe pain at 2 years for a baseline risk of $5.05 \%$. Patients with prefracture hip pain (OR 2.52; 95\% CI 1.25-5.06; ARI 12.5\%, 95\% CI 2.85\%-22.12\%; $P=$ 0.01 ), prefracture opioid use (OR 4.32; 95\% CI 2.08-8.95; ARI $21.1 \%$; 95\% CI 8.23\%-34.02\%; $P<0.001$ ), aged $\leq 79$ year-old (OR 1.92; 95\% CI 1.09-3.39; ARI 6.3\%; 95\% CI $2.67 \%-9.91 \% ; P=0.025)$, and who ambulated without assistive devices before surgery (OR 2.38; 95\% CI 1.28-4.44; ARI $10.7 \%$; 95\% CI 3.80\%-17.64\%; $P=0.006)$ were more likely to report moderate-to-severe pain after 2 years (Table 3 ).

The interaction between prefracture opioid use and functional status was not statistically significant $(P=0.32)$.

TABLE 2. Factors Associated With Moderate-to-Severe Pain (vs. Mild or No Pain) at 12 Months in Patients With Femoral Neck Fractures

\begin{tabular}{|c|c|c|c|c|c|}
\hline Factor & Unadjusted OR (95\% CI) & $\boldsymbol{P}$ & Adjusted OR (95\% CI) & $P$ & ARI \%(95\% CI) \\
\hline \multicolumn{6}{|l|}{ Age } \\
\hline Above median $(>79$ years $)$ & Reference & & Reference & & \\
\hline Below median $(\leq 79$ years $)$ & $1.41(0.92-2.17)$ & 0.109 & $1.42(0.86-2.32)$ & 0.167 & - \\
\hline \multicolumn{6}{|l|}{ Sex } \\
\hline Male & Reference & & Reference & & \\
\hline Female & $1.91(1.09-3.35)$ & 0.023 & $1.85(1.0-3.45)$ & 0.049 & $6.2(3.53-8.84)$ \\
\hline \multicolumn{6}{|l|}{ BMI } \\
\hline$\leq 24.9$ & Reference & - & Reference & & \\
\hline $25-34.9$ & $1.09(0.71-1.67)$ & 0.691 & $0.96(0.6-1.55)$ & 0.874 & - \\
\hline \multicolumn{6}{|l|}{$\begin{array}{l}\text { Prefracture moderate-to-severe hip } \\
\text { pain }\end{array}$} \\
\hline No & Reference & - & Reference & & \\
\hline Yes & $3.23(1.83-5.70)$ & $<0.001$ & $2.68(1.46-4.90)$ & 0.001 & $15.4(6.44-24.35)$ \\
\hline \multicolumn{6}{|l|}{ Prefracture opioid use } \\
\hline No & Reference & - & Reference & & \\
\hline Yes & $3.05(1.67-5.55)$ & $<0.001$ & $2.66(1.35-5.24)$ & 0.005 & $15.7(5.41-25.90)$ \\
\hline \multicolumn{6}{|l|}{ Prefracture functional status } \\
\hline Uses an assistive device & Reference & - & Reference & & \\
\hline Ambulate without assistance & $1.58(0.97-2.58)$ & 0.062 & $1.52(0.86-2.67)$ & 0.147 & - \\
\hline \multicolumn{6}{|l|}{ Depression } \\
\hline No & Reference & & Reference & & \\
\hline Yes & $1.53(0.85-2.73)$ & 0.152 & $1.19(0.6-2.33)$ & 0.62 & - \\
\hline \multicolumn{6}{|l|}{ Surgical approach } \\
\hline Posterior/posterolateral & Reference & - & Reference & & \\
\hline Anterolateral/direct anterior & $1.29(0.82-2.04)$ & 0.260 & $1.1(0.64-1.89)$ & 0.722 & - \\
\hline \multicolumn{6}{|l|}{ Surgery type } \\
\hline THA & Reference & - & Reference & & \\
\hline Bipolar hemiarthroplasty & $1.24(0.74-2.08)$ & 0.421 & $1.05(0.59-1.87)$ & 0.858 & - \\
\hline Unipolar hemiarthroplasty & $1.35(0.81-2.24)$ & 0.251 & $1.35(0.74-2.43)$ & 0.325 & - \\
\hline \multicolumn{6}{|l|}{ Weight-bearing status } \\
\hline Full & Reference & - & Reference & & \\
\hline Non or partial & $0.88(0.57-1.36)$ & 0.573 & $0.94(0.57-1.57)$ & 0.826 & - \\
\hline \multicolumn{6}{|c|}{ Early physiotherapy or rehabilitation } \\
\hline No & Reference & - & Reference & & \\
\hline Yes & $0.62(0.30-1.26)$ & 0.191 & $0.9(0.39-2.07)$ & 0.809 & - \\
\hline \multicolumn{6}{|l|}{ Serious adverse events } \\
\hline No & Reference & - & Reference & & \\
\hline Yes & $1.05(0.62-1.79)$ & 0.828 & $0.76(0.4-1.43)$ & 0.388 & - \\
\hline
\end{tabular}

Adjusted model: the Hosmer-Lemeshow (HL) test $P$ value: 0.833 ; C-statistics: 0.67.

$\mathrm{AR}$, absolute risk increase; BMI, body mass index; OR, odds ratio; THA, total hip arthroplasty. 
TABLE 3. Factors Associated With Moderate-to-Severe Pain (vs. Mild or No Pain) at 24 Months in Patients With Femoral Neck Fractures

\begin{tabular}{|c|c|c|c|c|c|}
\hline Factor & Unadjusted OR (95\% CI) & $P$ & Adjusted OR (95\% CI) & $P$ & ARI \% $(95 \% \mathrm{CI})$ \\
\hline \multicolumn{6}{|l|}{ Age } \\
\hline Above median ( $>79$ years) & Reference & & Reference & & \\
\hline Below median ( $\leq 79$ years) & $1.49(0.93-2.39)$ & 0.093 & $1.92(1.09-3.39)$ & 0.025 & $6.2(2.67-9.90)$ \\
\hline Male & Reference & & Reference & & \\
\hline Female & $1.15(0.66-2.00)$ & 0.615 & $1.20(0.63-2.28)$ & 0.561 & - \\
\hline \multicolumn{6}{|l|}{ BMI } \\
\hline \multicolumn{6}{|l|}{$\begin{array}{l}\text { Prefracture moderate-to-severe hip } \\
\text { pain }\end{array}$} \\
\hline No & Reference & & Reference & & \\
\hline Yes & $3.93(2.11-7.35)$ & $<0.001$ & $2.52(1.25-5.06)$ & 0.01 & $12.4(2.85-22.11)$ \\
\hline \multicolumn{6}{|l|}{ Prefracture opioid use } \\
\hline Ambulate without assistance & $2.32(1.39-3.87)$ & 0.001 & $2.38(1.28-4.44)$ & 0.006 & $10.7(3.80-17.63)$ \\
\hline \multicolumn{6}{|l|}{ Depression } \\
\hline No & Reference & & Reference & & \\
\hline Yes & $1.20(0.61-2.36)$ & 0.606 & $0.91(0.41-1.99)$ & 0.807 & - \\
\hline \multicolumn{6}{|l|}{ Surgical approach } \\
\hline Posterior/posterolateral & Reference & & Reference & & \\
\hline Anterolateral/direct anterior & $0.99(0.61-1.59)$ & 0.972 & $0.88(0.49-1.58)$ & 0.658 & - \\
\hline \multicolumn{6}{|l|}{ Surgery type } \\
\hline THA & Reference & & Reference & & \\
\hline Bipolar hemiarthroplasty & $1.58(0.90-2.75)$ & 0.108 & $1.42(0.76-2.66)$ & 0.27 & - \\
\hline Unipolar hemiarthroplasty & $1.59(0.89-2.80)$ & 0.112 & $1.97(1-3.88)$ & 0.052 & - \\
\hline \multicolumn{6}{|l|}{ Weight-bearing status } \\
\hline Yes & $1.14(0.63-2.04)$ & 0.655 & $1.06(0.54-2.08)$ & 0.87 & - \\
\hline
\end{tabular}

Adjusted model: the Hosmer-Lemeshow (HL) test $P$ value: 0.229 ; C-statistics: 0.71 .

$\mathrm{AR}$, absolute risk increase; BMI, body mass index; OR, odds ratio; THA, total hip arthroplasty.

We did not find any variables with a variance inflation factor greater than 1.50, and no variables had a deviance residual more than 3, which may suggest no evidence of serious multicollinearity or influential observations in our data.

\section{DISCUSSION}

We found that approximately one in 10 hip fracture patients experienced moderate-to-severe pain 2 years after arthroplasty. Younger age, female sex, higher functioning before fracture, living with hip pain, and use of prescription opioids were predictive of persistent pain after surgery.
In our adjusted analyses, patients reporting pain and use of opioids before their fracture occurred were more than twice as likely to report persistent moderate-to-severe pain after hip surgery, which is consistent with findings of other studies exploring prognosis after THA and total knee arthroplasty. ${ }^{21,22}$ Evidence suggests that chronic use of opioids may produce opioid-induced hyperalgesia, ${ }^{23}$ which can result in pain sensitivity and in developing persistent pain after surgery. ${ }^{24,25}$ In addition, opioids are commonly prescribed for chronic pain; pre-existing pain in another part of the body may influence pain severity of replaced joints. ${ }^{26,27}$ As such, there is a possibility that patients reported persistent 
moderate-to-severe pain after surgery because of an underlying chronic pain condition. ${ }^{28}$

Our results did not indicate that surgery type (eg, THA vs. bipolar or unipolar hemiarthroplasty) is a significant predictor of pain at 1 and 2 years after surgery. We also found no association between receiving early physiotherapy/ rehabilitation or weight-bearing status and pain at 12- and 24month follow-up. Consistent with our findings, a retrospective study did not find a significant difference between a full versus no weight-bearing status and pain among hip fracture patients who were treated with surgery, ${ }^{29}$ although other studies have shown that no weight-bearing status is related to poor functioning after surgery. ${ }^{30,31}$ Our findings also revealed that patients who were able to ambulate without assistive devices experienced more moderate-to-severe pain 2 years after hip arthroplasty. However, these results are in contrast to some studies that reported loss in long-term functional ability was inversely associated with higher prefracture functional status. ${ }^{31,32}$ These results suggest that the complex relationship between pain and function after hip fracture surgery requires further evaluation.

Female sex was also associated with persistent hip pain 1 year after surgery. Other researchers have reported similar findings and have stated that female sex is related to increased chronic pain, ${ }^{33,34}$ whereas other research has found no association. ${ }^{35}$ Younger ages ( $\leq 79$ vs. $>79$ years) predicted more moderate-to-severe pain 2 years after hip arthroplasty, which is consistent with previous studies that assessed predictors of chronic pain after surgery. ${ }^{36-38}$ Explanations for this result may relate to higher expectations of recovery among younger patients or biological differences, with younger patients probably having greater neuron system responsiveness as compared to older patients. ${ }^{39}$ Nevertheless, these findings differ from some studies ${ }^{40,41}$ that failed to show age as a significant predictor of persistent pain after surgery.

Our study has some limitations, including a high rate of incomplete data for the outcome, especially at the 2-year follow-up; however, our rate of missing data is consistent with previous studies in this area. ${ }^{42,43}$ Baseline characteristics of patients with loss to follow-up data for the outcome were similar to patients with complete data for all significant predictors except for prefracture hip pain and functional status related to the 24-month visit (see Table 1, Supplemental Digital Content 1, http://links.lww.com/JOT/B205). Some relevant comorbidities (eg, anxiety and substance use disorder) or the indication and dose of opioids that patients were using before were not collected in the trial, but for future studies, these variables should be considered. The strengths of our study include using a large, international, representative sample that increases the generalizability of our findings, investigating several clinically relevant independent variables in the prediction model, and using a validated instrument for pain assessment.

\section{CONCLUSION AND FUTURE RECOMMENDATIONS}

The results of this study provide insights into those at a higher risk of having moderate-to-severe pain at one and 2 years after hip arthroplasty. Patients with prefracture hip pain, opioid use, independent ambulatory status, female sex, and younger ages ( $\leq 79$ vs. greater 79 years) are more likely to have long-term moderate-to-severe pain after arthroplasty surgery for management of femoral neck fractures. This information will further inform health care providers and patients and allow for better understanding of the expected benefits of hip arthroplasty in this patient population.

\section{Acknowledgments}

The authors thank the HEALTH Investigators (http:// links.lww.com/JOT/B233).

\section{REFERENCES}

1. Carnevale V, Fontana A, Scillitani A, et al. Incidence and all-cause mortality for hip fracture in comparison to stroke, and myocardial infarction: a fifteen years population-based longitudinal study. Endocrine. 2017;58:320-331.

2. Clemens KK, Ouedraogo A, Speechley M, et al. Hip fractures in older adults in Ontario, Canada - monthly variation, insights, and implications. Can Geriatr J. 2019;22:148.

3. Peeters CM, Visser E, Van de Ree CL, et al. Quality of life after hip fracture in the elderly: a systematic literature review. Injury. 2016;47: 1369-1382.

4. Jongjit J, Komsopapong L, Songjakkaew P, et al. Health-related quality of life after hip fracture in the elderly community-dwelling. Southeast Asian J Trop Med Public Health. 2003;34:670-674.

5. Bentler SE, Liu L, Obrizan M, et al. The aftermath of hip fracture: discharge placement, functional status change, and mortality. Am J Epidemiol. 2009;170:1290-1299.

6. Roche J, Wenn RT, Sahota O, et al. Effect of comorbidities and postoperative complications on mortality after hip fracture in elderly people: prospective observational cohort study. BMJ. 2005;331:1374.

7. Brauer CA, Coca-Perraillon M, Cutler DM, et al. Incidence and mortality of hip fractures in the United States. JAMA. 2009;302:1573-1579.

8. Bhandari M, Devereaux P, Tornetta P III, et al. Operative management of displaced femoral neck fractures in elderly patients: an international survey. J Bone Joint Surg Am. 2005;87:2122-2130.

9. Kurtz SM, Lau E, Ong K, et al. Future young patient demand for primary and revision joint replacement: national projections from 2010 to 2030 . Clin Orthop Relat Res. 2009;467:2606-2612.

10. Yoo JI, Ha YC, Lim JY, et al. Early rehabilitation in elderly after arthroplasty versus internal fixation for unstable intertrochanteric fractures of femur: systematic review and meta-analysis. J Korean Med Sci. 2017;32: 858-867.

11. Ravikumar KJ, Marsh G. Internal fixation versus hemiarthroplasty versus total hip arthroplasty for displaced subcapital fractures of femur-13 year results of a prospective randomised study. Injury. 2000;31:793-797.

12. Liao L, min Zhao J, Su W, et al. A meta-analysis of total hip arthroplasty and hemiarthroplasty outcomes for displaced femoral neck fractures. Arch Orthop Trauma Surg. 2012;132:1021-1029.

13. Bhandari M, Einhorn TA, Guyatt G, et al. Total hip arthroplasty or hemiarthroplasty for hip fracture. N Engl J Med. 2019;381:2199-2208.

14. Burgers PT, Poolman RW, Van Bakel TM, et al. Reliability, validity, and responsiveness of the Western Ontario and McMaster Universities Osteoarthritis Index for elderly patients with a femoral neck fracture. $J$ Bone Joint Surg Am. 2015;97:751-757.

15. McConnell S, Kolopack P, Davis AM. The Western Ontario and McMaster Universities Osteoarthritis Index (WOMAC): a review of its utility and measurement properties. Arthritis Rheum. 2001;45:453-461.

16. Kapstad H, Hanestad BR, Langeland N, et al. Cutpoints for mild, moderate and severe pain in patients with osteoarthritis of the hip or knee ready for joint replacement surgery. BMC Musculoskelet Disord. 2008;9: 55 .

17. Goggins J, Baker K, Felson D. What WOMAC pain score should make a patient eligible for a trial in knee osteoarthritis? J Rheumatol. 2005;32: $540-542$. 
18. Xu BY, Yan S, Low LL, et al. Predictors of poor functional outcomes and mortality in patients with hip fracture: a systematic review. $B M C$ Musculoskelet Disord. 2019;20:568.

19. Hosmer DW, Hosmer T, Le Cessie S, et al. A comparison of goodness-offit tests for the logistic regression model. Statistics Med. 1997;16:965-980.

20. Akinwande MO, Dikko HG, Samson A. Variance inflation factor: as a condition for the inclusion of suppressor variable (s) in regression analysis. Open J Statistics. 2015;5:754.

21. Morris BJ, Sciascia AD, Jacobs CA, et al. Preoperative opioid use associated with worse outcomes after anatomic shoulder arthroplasty. $J$ Shoulder Elbow Surg. 2016;25:619-623.

22. Rakel BA, Blodgett NP, Zimmerman MB, et al. Predictors of postoperative movement and resting pain following total knee replacement. Pain. 2012;153:2192-2203.

23. Kum E, Buckley N, de Leon-Casasola O, et al. Attitudes towards and management of opioid-induced hyperalgesia: a survey of chronic pain practitioners. Clin J Pain. 2020;36:359-364.

24. Mercadante S, Ferrera P, Villari P, et al. Hyperalgesia: an emerging iatrogenic syndrome. J Pain Symptom Manage. 2003;26:769-775.

25. Angst MS, Clark JD. Opioid-induced hyperalgesia: a qualitative systematic review. Anesthesiology. 2006;104:570-587.

26. Nikolajsen L, Brandsborg B, Lucht U, et al. Chronic pain following total hip arthroplasty: a nationwide questionnaire study. Acta Anaesthesiol Scand. 2006;50:495-500.

27. Rat AC, Guillemin F, Osnowycz G, et al. Total hip or knee replacement for osteoarthritis: mid-and long-term quality of life. Arthritis Care Res. 2010;62:54-62.

28. Croft P, Dunn KM, Von Korff M. Chronic pain syndromes: you can't have one without another. Pain. 2007;131:237-238.

29. Baer M, Neuhaus V, Pape HC, et al. Influence of mobilization and weight bearing on in-hospital outcome in geriatric patients with hip fractures. SICOT J. 2019;5:4.

30. Ariza-Vega P, Jiménez-Moleón JJ, Kristensen MT. Non-weight-bearing status compromises the functional level up to $1 \mathrm{yr}$ after hip fracture surgery. Am J Phys Med Rehabil. 2014;93:641-648.

31. Mariconda M, Costa GG, Cerbasi S, et al. Factors predicting mobility and the change in activities of daily living after hip fracture: a 1-year prospective cohort study. J Orthop Trauma. 2016;30:71-77.
32. McGilton KS, Chu CH. Factors influencing outcomes of older adults after undergoing rehabilitation for hip fracture. $J$ Am Geriatr Soc. 2016;64:1601-1609.

33. Strauss A, Rommelspacher Y, Nouri B, et al. Long-term outcome of total hip arthroplasty in patients with haemophilia. Haemophilia. 2017;23: 129-134.

34. Sylliaas H, Thingstad P, Wyller TB, et al. Prognostic factors for self-rated function and perceived health in patient living at home three months after a hip fracture. Disabil Rehabil. 2012;34:12251231

35. MacWilliam CH, Yood MU, Verner JJ, et al. Patient-related risk factors that predict poor outcome after total hip replacement. Health Serv Res. 1996;31:623.

36. Nilsdotter AK, Lohmander LS. Age and waiting time as predictors of outcome after total hip replacement for osteoarthritis. Rheumatology. 2002;41:1261-1267.

37. Röder C, Parvizi J, Eggli S, et al. Demographic factors affecting longterm outcome of total hip arthroplasty. Clin Orthop Relat Res. 2003;6273.

38. Kessler S, Mattes T, Cakir B, et al. The impact of preoperative function and pain on early patient-centred outcome after total hip arthroplasty. $Z$ Orthop Unfall. 2007;145:563-567.

39. Gibson SJ, Helme RD. Age-related differences in pain perception and report. Clin Geriatr Med. 2001;17:433-456. v-vi.

40. Pagé MG, Katz J, Escobar EMR, et al. Distinguishing problematic from nonproblematic postsurgical pain: a pain trajectory analysis after total knee arthroplasty. Pain. 2015;156:460-468.

41. Sieberg CB, Klajn J, Wong C, et al. Predictors and trajectories of chronic postoperative pain following hip preservation surgery. J Hip Preserv Surg. 2017;4:45-53.

42. Riddle DL, Wade JB, Jiranek WA, et al. Preoperative pain catastrophizing predicts pain outcome after knee arthroplasty. Clin Orthop Relat Res. 2010;468:798-806.

43. Roth ML, Tripp DA, Harrison MH, et al. Demographic and psychosocial predictors of acute perioperative pain for total knee arthroplasty. Pain Res Manag. 2007;12:185-194. 